\title{
An Online Energy Management Strategy for a Fuel Cell/Battery Vehicle Considering the Driving Pattern and Performance Drift Impacts
}

\author{
M. Kandidayeni, Student Member, IEEE, A. Macias, Student Member, IEEE, A. Khalatbarisoltani, \\ Student Member, IEEE, L. Boulon, Senior Member, IEEE, S. Kelouwani, Senior Member, IEEE, H. \\ Chaoui, Senior Member, IEEE
}

\begin{abstract}
Energy management strategy (EMS) has a profound influence over the performance of a fuel cell hybrid electric vehicle since it can maintain the energy sources in their high efficacy zones leading to efficiency and lifetime enhancement of the system. This paper puts forward an online multi-mode EMS to efficiently split the power among the components while embracing the effects of the driving conditions and performance degradation of the fuel cell system. In this regard, firstly, a self-organizing map (SOM) is trained to cluster the driving patterns. The SOM competitive layer in this work is composed of ten driving features as inputs and it classifies the driving patterns into three classes in the output. Subsequently, a three-mode fuzzy logic controller (FLC) is designed and optimized offline by the genetic algorithm for each driving pattern. Unlike the other similar works, the output membership function of the FLC is designed based on the online identification of the maximum power and efficiency of the fuel cell system which change over time. Finally, the SOM is utilized to recognize the driving mode at each sequence and accordingly activate the most suitable mode of the FLC to meet the requested power by efficient use of the energy sources. The performance of the proposed EMS has been validated by using the hardware-in-the-loop platform for several scenarios. The experimental results analyses indicate the promising performance of the suggested methodology in terms of ameliorating hydrogen economy and the fuel cell system lifetime.
\end{abstract}

Index Terms-Driving condition prediction, fuel cell hybrid electric vehicle, fuzzy logic control, PEMFC online parameter estimation, self-organizing map.

\section{INTRODUCTION}

$\mathrm{T}$ RANSPORTATION is broadly held responsible for producing carbon dioxide emissions resulting from the burning of

This work was supported in part by the Natural Sciences and Engineering Research Council of Canada (NSERC), the Fonds de recherche du Québec Nature et technologies (FRQNT), and Canada Research Chairs program.

M. Kandidayeni, A. Macias, and A. Khalatbarisoltani are with the Hydrogen Research Institute, Department of Electrical and Computer Engineering, Université du Québec à Trois-Rivières, QC G8Z 4M3, Canada (email: mohsen.kandi.dayeni@uqtr.ca, alvaro.omar.macias.fernandez@uqtr.ca , and arash.khalatbarisoltani@uqtr.ca).

L. Boulon is with the Hydrogen Research Institute, Department of Electrical and Computer Engineering, Université du Québec à Trois-Rivières, QC G8Z 4M3, Canada (e-mail: loic.boulon@uqtr.ca ).

S. Kelouwani is with the Hydrogen Research Institute, Department of Mechanical Engineering, Université du Québec à Trois-Rivières, QC G9A 5H7, Canada (e-mail: sousso.kelouwani@uqtr.ca).

H. Chaoui is with the Intelligent Robotic and Energy Systems Research Group, Department of Electronics, Carleton University, Ottawa, ON K1S 5B6, Canada (e-mail: hicham.chaoui@carleton.ca). fossil fuels, such as gasoline, in internal combustion engines [1]. Pure electric and hybrid electric vehicles have been thought-provoking transitional alternates for conventional vehicles although the latter is still dependent on fossil fuels and the former has limited driving range in addition to long recharging time [2]. These shortfalls have also provided the basis for the advent of new power sources such as proton exchange membrane (PEM) fuel cells (FCs) in vehicular applications, which are presenting a steadily growing division of the automotive market [3]. Fuel cell hybrid electric vehicles (FCHEVs) usually utilize a PEMFC as the primary power source and a battery pack or/and a supercapacitor as the secondary power source. Therefore, the performance of an FCHEV is impacted by several interrelated factors which put the design of an energy management strategy (EMS) in critical position [4]. Regardless of the type of the hybrid vehicle, the existing EMSs fall under two categories of rule-based and optimization-based [5, 6]. The rule-based strategies are usually heuristic and lead to limited and sub-optimal solutions. In this regard, the researchers have turned attentions on the optimization methods, which assure optimal or near-optimal solutions in theory and can also provide new guidelines for refining the rule-based methods [7,8]. Optimization-based strategies can be divided into two groups of global and real-time strategies depending on the defined cost function. The former utilizes the cost function over a fixed driving cycle and is beneficial for realizing the optimal policy. However, it is not applicable in real-time control of the vehicle owing to its dependency on the driving profile. The latter, nonetheless, uses an instantaneous cost function based on the variables of the system. Equivalent consumption minimization strategy (ECMS) and Pontryagin's minimum principle (PMP) are two widely used real-time optimization strategies in hybrid electric vehicles [9-12]. One of the key issues here is the high instantaneous computational time. Furthermore, the estimation of the equivalent factor in ECMS and the initialization of the co-state in PMP, which are sensitive to transient dynamic and the driving pattern, are quite challenging tasks $[13,14]$.

In light of the discussed matters, the use of traffic condition and driving information in the design of an EMS has come under the attention of many researchers [15-17]. This line of work is known as intelligent-based EMS category and can be integrated into both of ruled-based and optimization-based strategies [18]. Intelligent-based EMSs mainly consist in the use of car navigation data (global positioning system, vehicle 
geographical information system or vehicle telematics) and the history of motion for recognizing and predicting the driving condition [19]. Several approaches based on fuzzy logic control (FLC) [20], neural networks (NNs) [21], and other machine learning-based techniques [22] have been introduced in this respect. In [23], the driving data is clustered by using a hierarchical algorithm and support vector machine (SVM) is used for the recognition of the traffic condition. In [24], multilayer perceptron (MLP) NN is trained to recognize the driving pattern and activates the controller. In [25], back propagation $\mathrm{NN}$ along with metaheuristic algorithms is used to formulate a dynamic programming-based predictive EMS to reduce the fuel consumption. In [26], the suggested strategy comprises two steps: generation of optimal EMS for the long trip by using an estimation of distribution optimization algorithm and refining the optimal EMS with regard to actual traffic conditions in the short-term. In [27], combination of PMP with NN, in [28], learning vector quantization $\mathrm{NN}$ with $\mathrm{GA}$, and in [29], probabilistic SVM with a data fusion based method are proposed for developing the EMS. In [30], an adaptive control based on tuning the FLC parameters for different loads is proposed. The authors state that the PEMFC voltage declines due to degradation after a while and under this condition the rule-based values should be reconsidered.

Apart from the importance of considering the driving condition, it is also essential to take into account the performance drifts of the FC system in EMS formulation of an FCHEV. The performance of a FC system is impacted by several factors such as the variation of operation conditions and degradation phenomenon. The previous works of the authors have touched upon the procedure for updating the parameters of a FC system online [31, 32]. However, they have not been integrated into the EMS design yet. There are some works in the literature regarding the online identification of the PEMFC model in an EMS. Some of them are based on the extremum seeking methods in which a periodic perturbation signal is utilized to find an optimal operating point in real-time [33-35]. Such strategies are employed in the formulation of an EMS mainly due to their easy implementation. However, they cannot be very effective when simultaneous identification of several operating points are required in online applications. Because they need a separate search line for each intended characteristic such as maximum efficiency (ME) and maximum power (MP). This problem can be avoided by utilizing recursive filters for online identification of the PEMFC parameters and extracting the necessary characteristics from the updated model. There are a few EMSs on this basis in the literature for FCHEVs. In [36, 37], the authors employ the recursive least square (RLS) and the square root unscented Kalman filter (KF) for updating a single-input (current) PEMF model while designing hysteresis and PMP based EMSs for a FCHEV. They indicate that the classical EMSs are not very efficient when there are drifts in the FC system. In [38], the authors propose a supervisory controller while the PEMFC model is being updated by a simple current dependent model.

This paper proposes a novel adaptive soft-computing based EMS for a FCHEV, composed of a FC system and a battery pack. This is one of the first attempts, if any, to merge both of driving pattern recognition and adaptation to the performance drifts of the FC system in a single EMS. The core of the suggested strategy is an online self-organizing map (SOM) driving profile classifier and a multi-mode FLC with online updating of the output defuzzification. To the best of the authors' knowledge, SOM has not already been used as a driving condition recognition tool. Moreover, the other contribution of this work lies in the formulation of the FLC to adapt to the real state of the FC system. Each FLC has three inputs including requested power, derivation of requested power, and battery state of charge (SOC), and one output, which determines the portion of required power form the PEMFC system. The defuzzification of the FLC output is done based on the estimation of MP and ME of the real FC system through an online model composed of a PEMFC semi-empirical model coupled with KF. Contrary to most of the existing papers in the literature which are based on simulation, the obtained results of this work have been validated on a developed test bench by using hardware-in-the-loop (HIL) technique. To highlight the influence of tracing the real state of a FC system while designing an EMS, two PEMFCs with different degrees of degradation are used in the experimental section of this paper.

Section II deals with the modeling description of the vehicle. The methodology for designing the proposed EMS is detailed in Section III. Section IV clarifies the obtained results from different considered scenarios. Finally, the conclusion along with some remarks is presented in section $\mathrm{V}$.

\section{FUEL CELL HYBRID ELECTRIC VEHICLE SYSTEM}

\section{A. Hardware-in-the-loop platform and Power train system modeling}

The system used in this work is based on a low-speed FCHEV called Nemo. The main characteristics of the vehicle are listed in Table I.

TABLE I

NEMO PARAMETERS DEFINITION

\begin{tabular}{ccc}
\hline & NEMO PARAMETERS DEFINITION & \\
\hline Specification & Parameter & Value \\
& Rolling resistance & 0.015 \\
& Aerodynamic drag & 0.42 \\
& Frontal area $\left(\mathrm{m}^{2}\right)$ & 4 \\
& Density of air $\left(\mathrm{kg} / \mathrm{m}^{3}\right)$ & 1.2 \\
Vehicle's parameters & Mass factor & 1.035 \\
& Mass $(\mathrm{kg})$ & 896 \\
& Maximum speed & 40 \\
3-phase induction & (km/h) & 5690 \\
machine & Power $(\mathrm{W})$ & 131.1 \\
FC system & Frequency $(\mathrm{Hz})$ & 4 \\
& Rated power $(\mathrm{kW})$ & 72 \\
Battery & voltage $(\mathrm{V})$ & 120 \\
& Capacity $(\mathrm{Ah})$ &
\end{tabular}

For the purpose of this paper, a HIL set-up, as shown in Fig. 1, is designed for evaluating the performance of the EMS. The FC system is the real component of this HIL simulator and the other ones are the mathematical models. In this set-up, a Horizon 500-W air breathing PEMFC, which is connected to a National Instrument CompactRIO through its controller, is utilized. The FC controller controls the mounted axial fan which is responsible for cooling the stack and supplying the necessary oxygen. The information between the CompactRIO 
and the PC is transferred by an Ethernet connection every 100 milliseconds. Temperature, current, and voltage of the FC system are recorded and used for the online modeling. An 8514 BK Precision DC Electronic Load is used to request the load profile, imposed by the DC-DC converter, from the FC system. According to Table I, the Nemo FCHEV has a 4-kW FC system. In this regard, the FC output voltage in the HIL set-up is scaled up after the converter to meet the requested power. To emphasize the significance of tracking the real behavior of the FC system while designing an EMS, two H-500 Horizon PEMFCs with different degrees of degradation are used in this work. The MP and ME curves of each of the FC systems are presented in Fig. 2 and their other characteristics are listed in Table II. Hereafter in this manuscript, the degraded PEMFC is called old PEMFC and the other one is referred to as new PEMFC. Fig. 2 also shows the safe zone operating range, between the ME and MP boundaries, of these FC systems. The EMSs should try to operate the PEMFC in this safe zone to increase the lifetime and fuel economy of the system. It should be noted that according to [37], a dynamic limitation of 50 $\mathrm{Ws}^{-1}$, which means a maximum of $10 \%$ of the maximum power per second for rising, and also $30 \%$ of the maximum power per second for falling, as suggested in [39], have been considered for the operation of the PEMFC stack.

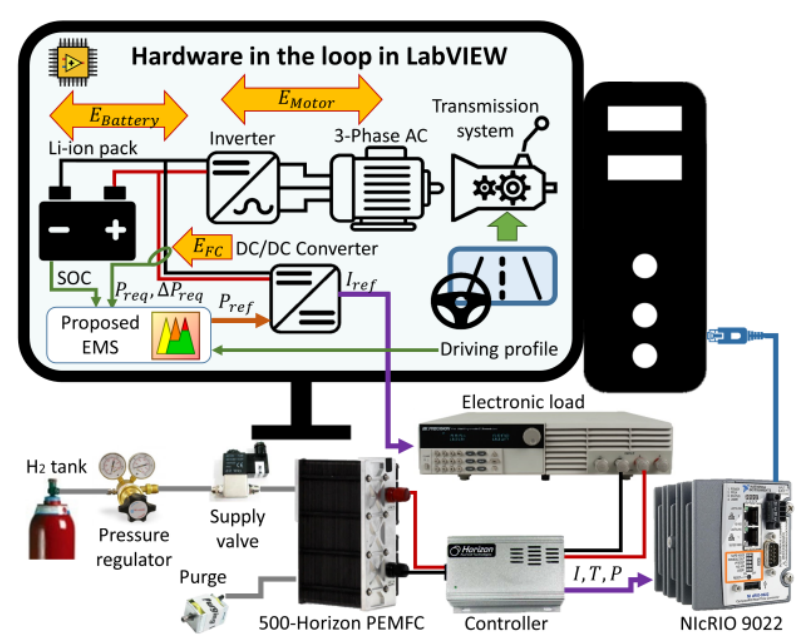

Fig. 1. The HIL set-up for testing the EMS.

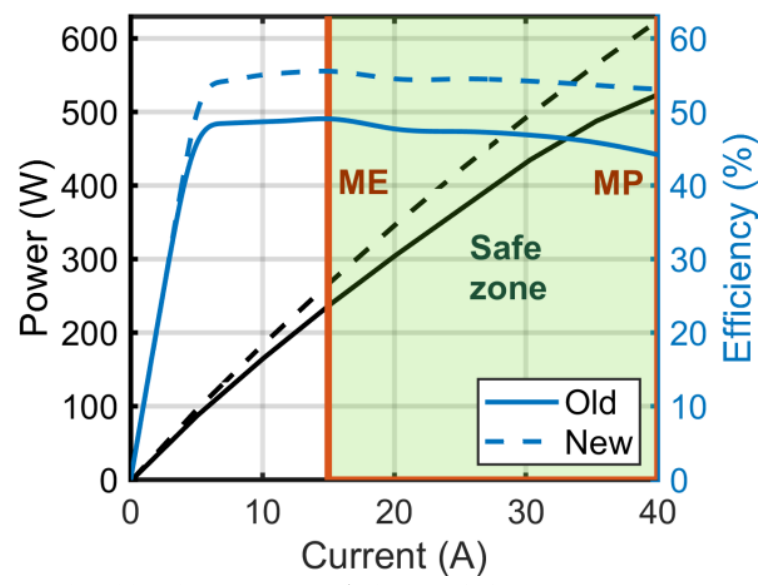

Fig. 2. The old and new PEMFCs' characteristics.
TABLE II

THE TECHNICAL FEATURES OF THE H-500 HORIZON PEMFC

\begin{tabular}{ll}
\hline \hline \multicolumn{2}{c}{ PEMFC Technical specification } \\
\hline Type of FC & PEM \\
Number of cells & 36 \\
Active area & $56 \mathrm{~cm}^{2}$ \\
Max Current (shutdown) & $42 \mathrm{~A}$ \\
Hydrogen pressure & $50-60 \mathrm{kPa}(0.5-0.6 \mathrm{Bar})$ \\
Rated $\mathrm{H}_{2}$ consumption & $7 \mathrm{SLPM}$ \\
Ambient temperature & 5 to $30^{\circ} \mathrm{C}$ \\
Max stack temperature & $65^{\circ} \mathrm{C}$ \\
Cooling & Air (integrated cooling fan) \\
\hline \hline
\end{tabular}

According to Fig. 1, the FC system, as the primary power source, is connected to the DC bus via a DC-DC converter, and the battery pack, as the secondary source, is directly linked to the bus to sustain the voltage of the DC-link. The force of the hybrid vehicle, considering the speed $\left(V_{H V}\right)$ and mass $(m)$, can be calculated by taking into account the traction $\left(F_{t r}\right)$ and resistive $\left(F_{\text {res }}\right)$ forces as follows:

$\left\{\begin{array}{l}m \frac{d}{d t} V_{H V}=F_{t r}-F_{r e s} \\ F_{r e s}=m g f_{r}+0.5 \rho_{a} C_{d} A_{f} V^{2}+m g \alpha \\ F_{t r}=\left(V_{H V-r e f}-V_{H V}\right) C s(t)+F_{r e s}\end{array}\right.$

Where a family of PI controller is used to force the vehicle to follow the driving cycle and assure achieving the reference speed of the vehicle. The vehicle requested power from the electric motor side can be then expressed as:

$P_{\text {req }}=\left(V_{H V} \times F_{t r}\right) / \eta_{E M} \eta_{t} \eta_{d c-a c}$

Where $\eta_{t}$ is the transmission efficiency (92\%), $\eta_{E M}$ is the motor average efficiency $(90 \%)$, and $\eta_{d c-a c}$ is the inverter efficiency $(95 \%)$. A lithium-ion battery pack is used to help the FC stack to meet the energy demand from the electric motor side. The important parameters of the battery are listed in Table III. An internal resistance based model is used for modeling the behavior of this energy storage system [40]. Fig. 3 shows the relationship of battery SOC with each of open circuit voltage $\left(U_{b a t-O C}\right)$, internal resistance $\left(R_{b a t}\right)$ changes in charge, and internal resistance changes in discharge. The battery current $\left(I_{\text {bat }}\right)$, bus voltage $\left(U_{\text {bus }}\right)$, and SOC are calculated based on (3)(5) respectively.

$$
\begin{aligned}
& I_{b a t}=\frac{\left(U_{b a t-O C}-\sqrt{U_{b a t-O C}{ }^{2}-4 \times R_{b a t} \times P_{b a t}}\right.}{2 \times R_{b a t}} \\
& U_{b u s}=U_{b a t-O C}-I_{b a t} \times R_{b a t} \\
& \operatorname{SOC}\left(t_{f}\right)=\operatorname{SOC}\left(t_{0}\right)-\eta_{C} \frac{\int_{t_{0}}^{t} I_{b a t} d t}{C_{b a t}}
\end{aligned}
$$

where $P_{b a t}$ is the battery pack power, $C_{b a t}$ is the capacity, and $\eta_{C}$ is the coulombic efficiency.

The FC system modeling is premised on a semi-empirical equation proposed by Mann et al [41]. This model calculates the stack voltage for a number of cells connected in series. In fact, this model is being used for two purposes. 
TABLE III

THE UTILIZED BATTERY PACK DATA

\begin{tabular}{ccc}
\hline \hline Specification & Parameter & Value \\
\hline & Maximum current & $\mathrm{C} / 1 \mathrm{~A}$ \\
& continuous & $6 \mathrm{Ah}$ \\
CAFT Rechargeable & Nominal voltage & $3.65 \mathrm{~V}$ \\
lithium-ion battery cell & No. of cells in series & 20 \\
& No. of cells in parallel & 13 \\
& Cell mass & $0.34 \mathrm{~kg}$ \\
& Coulombic efficiency & 0.99 \\
\hline \hline
\end{tabular}

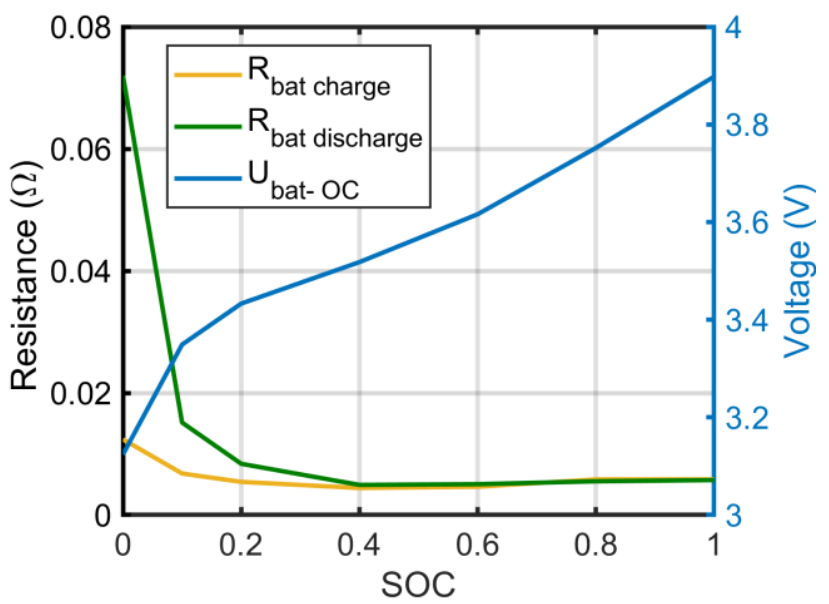

Fig. 3. The relationship of SOC with $U_{b a t-o C}$ and $R_{b a t}$ per cell.

First, for tuning the EMS parameters before its implementation on the real system to make sure that it does not damage the real FC system. Second, it is used in the online characteristics estimation process of the real FC stack while it is under operation.

$$
\begin{aligned}
& V_{F C}=N\left(E_{\text {Nernst }}+V_{\text {act }}+V_{\text {ohmic }}+V_{\text {con }}\right) \\
& E_{\text {Nernst }}=1.229-0.85 \times 10^{-3}(T-298.15)+4.3085 \times \\
& 10^{-5} T\left[\ln \left(P_{H_{2}}\right)+0.5 \ln \left(P_{O 2}\right)\right] \\
& \left\{V_{a c t}=\xi_{1}+\xi_{2} T+\xi_{3} T \ln \left(C O_{2}\right)+\xi_{4} T \ln \left(i_{F C}\right)\right. \\
& \left\{\mathrm{CO}_{2}=\frac{P_{O 2}}{5.08 \times 10^{-6} \exp (-498 / T)}\right. \\
& V_{\text {ohmic }}=-i_{F C} R_{\text {internal }}=-i_{F C}\left(\zeta_{1}+\zeta_{2} T+\zeta_{3} i_{F C}\right) \\
& V_{\text {con }}=B \ln \left(1-\frac{J}{J_{\max }}\right)
\end{aligned}
$$

Where $V_{F C}$ is the output voltage (V), $N$ is the number of cells, $E_{\text {Nernst }}$ is the reversible cell potential $(\mathrm{V}), V_{a c t}$ is the activation loss (V), $V_{\text {ohmic }}$ is the ohmic loss (V), $V_{\text {con }}$ is the concentration loss $(\mathrm{V}), T$ is the stack temperature $(\mathrm{K}), P_{H 2}$ is the hydrogen partial pressure in anode side $\left(\mathrm{N} \mathrm{m}^{-2}\right), P_{O 2}$ is the oxygen partial pressure in cathode side $\left(\mathrm{N} \mathrm{m}^{-2}\right), \xi_{n}(n=1 \ldots 4)$ are the semiempirical coefficients based on fluid mechanics, thermodynamics, and electrochemistry, $\mathrm{CO}_{2}$ is the oxygen concentration $\left(\mathrm{mol} \mathrm{cm}^{-3}\right), i_{F C}$ is the PEMFC operating current (A), $R_{\text {internal }}$ is the internal resistor $(\Omega), \zeta_{n}(n=1 \ldots 3)$ are the parametric coefficients, $B$ is a parametric coefficient $(\mathrm{V}), J$ is the actual current density $\left(\mathrm{A} \mathrm{cm}^{-2}\right)$, and $J_{\text {max }}$ is the maximum current density $\left(\mathrm{A} \mathrm{cm}^{-2}\right)$. The hydrogen flow is computed by a first order function approximation based on the experimental data, where $a$ and $b$ are fitting parameters [37].

$$
H_{2, \text { flow }}=a+b * i_{F C}
$$

The FC system is connected to the DC bus through a DC-DC converter. This converter is modeled by using a smoothing inductor and a boost chopper as formulated in (12) and (13). The detailed explanation of the converter model can be found in [42].

$L \frac{d}{d t} i_{F C}=V_{F C}-V_{h F C}-r_{L} i_{F C}$

$\left\{\begin{array}{c}V_{h F C}=m_{h f c} U_{b a t} \\ i_{h F C}=m_{h f c} i_{F C} \eta_{h F C}^{j}\end{array}\right.$ with $j=\left\{\begin{array}{c}1 \text { if } P_{c o n v}>0 \\ -1 \text { if } P_{c o n v}<0\end{array}\right.$

Where $L$ is the converter inductance $(\mathrm{H}), V_{h F C}$ is the input voltage in the chopper $(\mathrm{V}), r_{L}$ is the converter resistance $(\Omega)$, $m_{h f c}$ is the modulation ratio, and $\eta_{h F C}^{j}$ is the converter efficiency. In fact, the converter uses a voltage controller to determine the required coil current by minimizing the error between the actual and reference voltage of the FC system. Then a current controller is used to determine $m_{h f c}$ which boosts the output voltage to the desired value.

\section{ENERGY MANAGEMENT STRATEGY DESIGN}

The proposed EMS in this work aims at dealing with two important uncertainties, namely driving pattern changes and performance drifts of the FC system. The general structure of the suggested EMS is shown in Fig. 4. As is seen in this figure, the proposed online EMS comprises three important parts, namely traffic condition recognizer, online PEMFC modeling, and multi-mode FLC. The SOM is employed to determine the driving mode at each sequence and consequently trigger the most appropriate mode of the multi-mode FLC to satisfy the requested power. The updated characteristics of the online PEMFC stack model are also utilized to tune the output of the FLC with respect to the performance drifts of the PEMFC system. The development of each part is carefully described hereinafter.

\section{A. Traffic condition recognizer}

In this work, SOM, as an unsupervised learning method, is used for recognizing the driving condition. Kohonen presented this form of unsupervised competitive ANN in 1982 and it has been well studied and implemented in different problems [43]. In SOM, each of the neurons is associated to all the network inputs and to the neighborhood of the nearby neurons. Contrary to the utilized supervised methods, such as MLP and SVM, in driving condition recognition, SOM can update its weights without the need for a priori known labeled output. Compared to other unsupervised methods, like k-means and linear vector quantization, SOM preserves the structure of the original data, is very conducive to the interpretation of the clusters, and teaches the adjacent neurons to distinguish the neighboring sections of the input space. SOM has four phases of training as initialization, competition, cooperation, and adaptation.

In the training process, initially, each neuron is entirely linked to all the source nodes in the input vector and the weights of all the connections are assigned with small random values. 


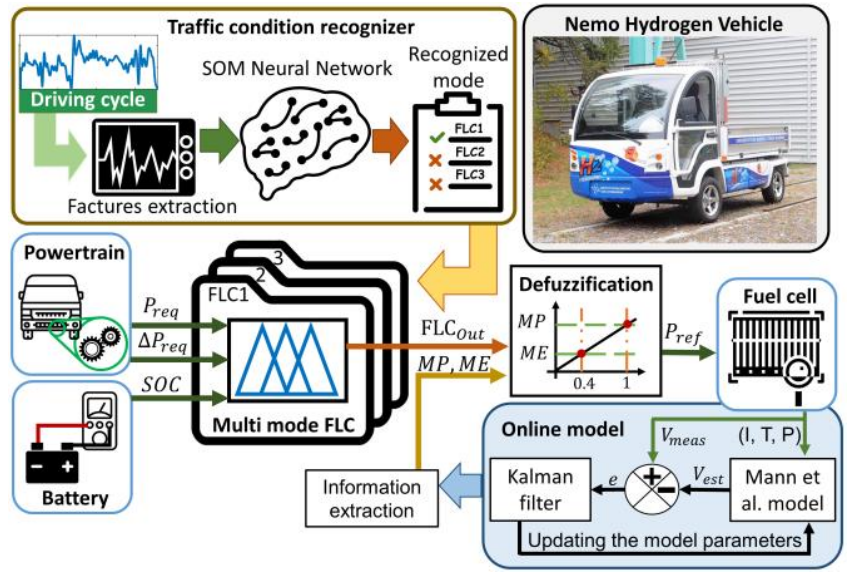

Fig. 4. The general architecture of the proposed online multi-mode EMS.

Then, the competition phase is performed by calculating the inner product between neuron j's weight vector $\left(W_{j}\right)$ and the input vector $(X)$. The winning neuron $(i(X))$ is determined by:

$$
\left\{\begin{array}{l}
i(X)=\underset{j}{\arg \min _{j}\left\|X-W_{j}\right\|} \\
\text { with }\left\{\begin{array}{l}
X=\left[X_{1}, X_{2}, \ldots, X_{m}\right]^{T} \\
W_{j}=\left[W_{j 1}, W_{j 2}, \ldots, W_{j m}\right]^{T}, j=1,2, \ldots, l
\end{array}\right.
\end{array}\right.
$$

where $m$ is the dimension of the input vector, and $l$ is the total number of neurons in the network. In the cooperation phase, the neurons in the neighborhood of the excited neuron are also tuned based on the principle of lateral interaction among the activated neurons of the human brain. This topological neighborhood is defined by:

$$
\left\{\begin{array}{l}
h_{j, i(X)}=\exp \left(-d_{j, i}^{2} / 2 \sigma^{2}\right) \\
\text { with }\left\{\begin{array}{l}
d_{j, i}^{2}=\left\|r_{j}-r_{i}\right\|^{2} \\
\sigma(n)=\sigma_{0} \exp \left(-n / \tau_{1}\right), n=0,1,2, \ldots,
\end{array}\right.
\end{array}\right.
$$

where $h_{j, i(X)}$ is the topological function, $d_{j, i}$ is the lateral distance, $\sigma$ is the standard deviation, $r_{j}$ is the position of the activated neuron, $r_{i}$ is the position of the wining neuron, $\sigma_{0}$ is the initial value of the $\sigma$, and $\tau$ is the time constant. Finally, in the adaptation phase, it is required that the weights of the wining neuron and its neighbors get updated. The weight adaptation is defined as:

$$
\left\{\begin{array}{l}
W_{j}(n+1)=W_{j}(n)+\eta(n) h_{j, i(X)}(n)\left(X(n)-W_{j}(n)\right) \\
\text { where }: \eta(n)=\eta_{0} \exp \left(-n / \tau_{2}\right), n=0,1,2, \ldots,
\end{array}\right.
$$

where $\eta(n)$ is the learning-rate parameter of the algorithm. The initial value of learning rate $\left(\eta_{0}\right)$ is usually defined as 0.1 and gradually decreases to around 0.01 . Once the SOM is trained, it can be used to classify the new input data based on the defined clusters.

In this manuscript, the input layer consists of ten neurons which are among ten important driving features introduced in $[23,28]$. Each of these neurons corresponds to one driving feature. Table IV lists the used features and driving schedules for SOM training. All the driving cycles have been scaled down according to the maximum speed of the Nemo vehicle, which is $40 \mathrm{~km} / \mathrm{h}$. In this way, the real driving patterns are best represented. The number of driving features in the input layer can vary from 2 to 62 in the literature. However, the chosen features of this work are among the most used ones. In order to train the SOM to classify the input features, twelve driving cycles are employed. Since SOM learns to classify the data based on how they are grouped in the input space, the driving cycles are chosen in a way to reach a homogeneous distribution of data. In other words, the chosen driving cycles cover all the driving conditions. To extract the statistical features, the driving cycles are decomposed into micro-trips and all the features are calculated for each micro-trip [20]. Concerning the number of output layer neurons, based on which the clusters are defined, Silhouette criterion is used. In this regard, SOM is trained for different number of output neurons $(2,3, \ldots, 6)$ by using the same input data. In each case, the Silhouette value is calculated for all the data of each cluster and its mean values are shown in Fig. 5. This value demonstrates how analogous an entity is to its own cluster (consistency) compared to other clusters (segregation). Silhouette value changes from -1 to +1 , where higher values denote that the entity fits well to its own cluster. According to Fig. 5, by grouping the driving data into three clusters, more cohesion clusters are attained. Therefore, three neurons are selected for the output layer of SOM to classify the driving data into three classes of slow-speed, medium-speed, and high-speed driving profiles.

TABLE IV

THE USED DRIVING FEATURES AND SCHEDULES

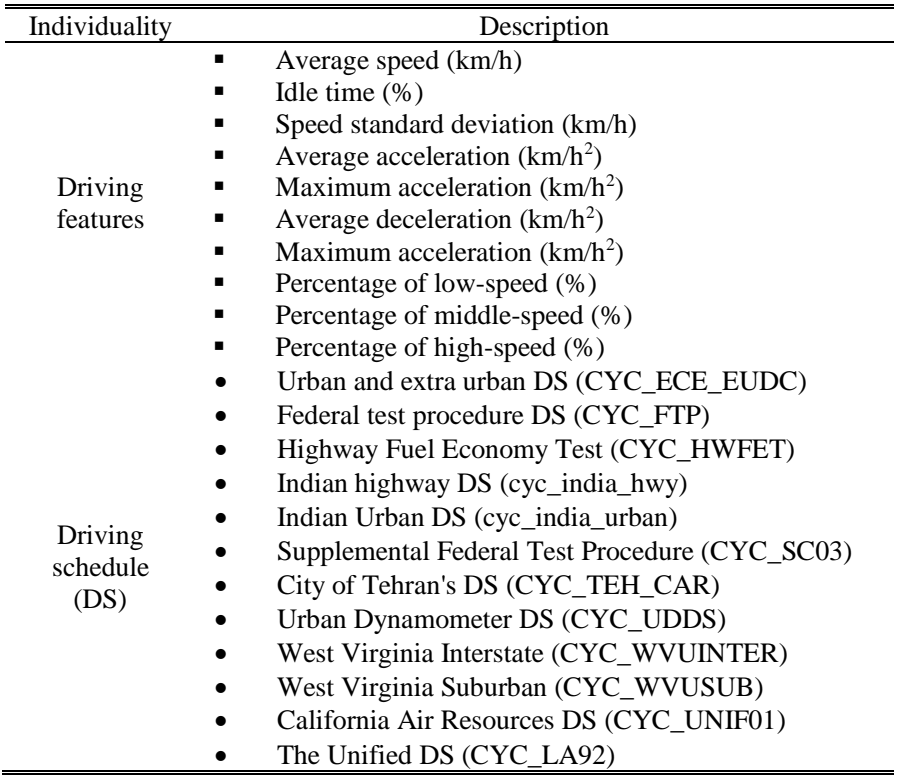

After training the SOM classifier, it can be used online for determining the driving conditions. In this respect, as suggested in [24], a sampling window size of $150 \mathrm{~s}$ and an updating window size of $50 \mathrm{~s}$ are employed to extract the statistical driving features while avoiding frequently mode switches. This means that each recognition is based on the driving features of 
the previous 150 -s window and is updated every 50 s. Fig. 6 demonstrates the 2D SOM weight positions based on average velocity and standard deviation (SD) in the initial phase and at the end of self-organization. As is seen in Fig. 6a, primary weights are scattered over the input space after the initialization. The red spots show the training vectors. However, after 1000 epochs, the neurons have moved towards the various training groups by updating the weights of the winner neurons and their neighbors according to Fig. $6 \mathrm{~b}$.

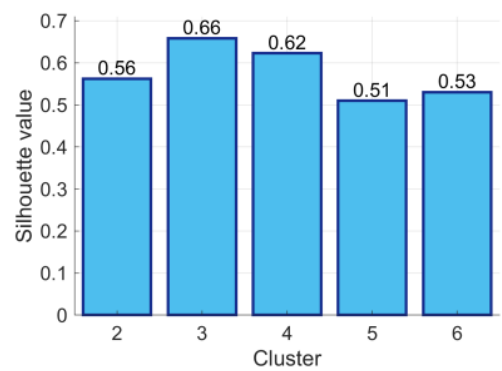

Fig. 5: The mean of Silhouette value for each cluster.
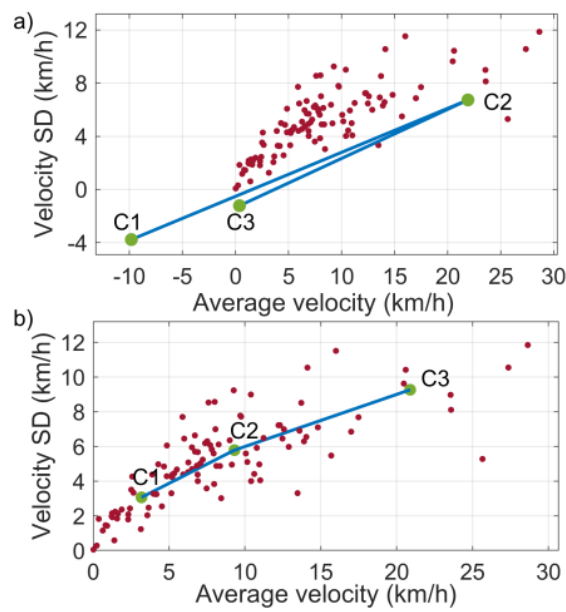

Fig. 6: The SOM based driving condition recognizer weight positions. (a): Initial weight positions, (b): The trained SOM weight positions.

A combined driving cycle, made up of CYC_NewYorkBus, CYC_UDDS, and worldwide harmonized light-duty vehicles test cycles (WLTC_class 3), is used to assess the performance of the SOM driving condition recognizer, as shown in Fig. 7. As it can be seen, the classifier is capable of recognizing new driving data without switching or confusing the conditions. It is worth noting that the CYC_NewYorkBus and WLTC_class 3 driving cycles are completely new for the classifier and have not been used in its training phase.

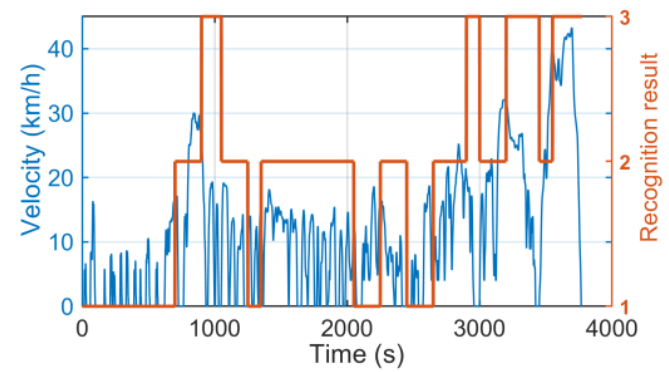

Fig. 7: Recognition results for the combined driving cycle.

\section{B. Fuel cell online modeling}

As previously mentioned, the parameters of a PEMFC model vary slowly over time since the device is affected by degradation and operating conditions. KF, as an optimal estimator, has been suggested for online parameter estimation of a FC system in the previous work of the authors [31]. KF can conclude the parameters of interest from imprecise and uncertain observations. This filter estimates the current state variables firstly and then updates them when the next measurement is received. The standard form of KF, introduced in [44], has been used in this paper. Table $\mathrm{V}$ defines some of the important parameters of KF in this work. The details about initialization and customization of $\mathrm{KF}$ for updating the parameters of the introduced PEMFC semi-empirical model, in section II, are comprehensively discussed in [31]. The ME and MP of the PEMFC are extracted from the updated model and used in the multi-mode FLC. Fig. 8 shows the capability of the online model in estimating the output voltage of the old FC system for the presented current and temperature profiles. Moreover, the hydrogen flow obtained by the model is compared with the measured one. Fig. 9 shows the predicted maximum power and efficiency curves of the old FC system.

TABLE V

KF CUSTOMIZATION FOR THE IDENTIFICATION PROBLEM

\begin{tabular}{|c|c|c|}
\hline Operators & SYMBOLS & Implementation description \\
\hline State vector & $x(t)$ & {$\left[\xi_{1}, \xi_{2}, \xi_{3}, \xi_{4}, \zeta_{1}, \zeta_{2}, \zeta_{3}, B\right]$} \\
\hline $\begin{array}{l}\text { Measurement } \\
\text { vector }\end{array}$ & $H(t)$ & $\begin{array}{l}{\left[1, T, T \ln \left(\mathrm{CO}_{2}\right), T \ln (i),-i,-i T,-\right.} \\
\left.i^{2}, \ln \left(1-\frac{J}{J_{\max }}\right)\right]\end{array}$ \\
\hline Transition matrix & $F(t+1 \mid t)$ & Identity matrix \\
\hline Measured output & $y(t)$ & Measured $V_{F C}$ from the real FC \\
\hline
\end{tabular}
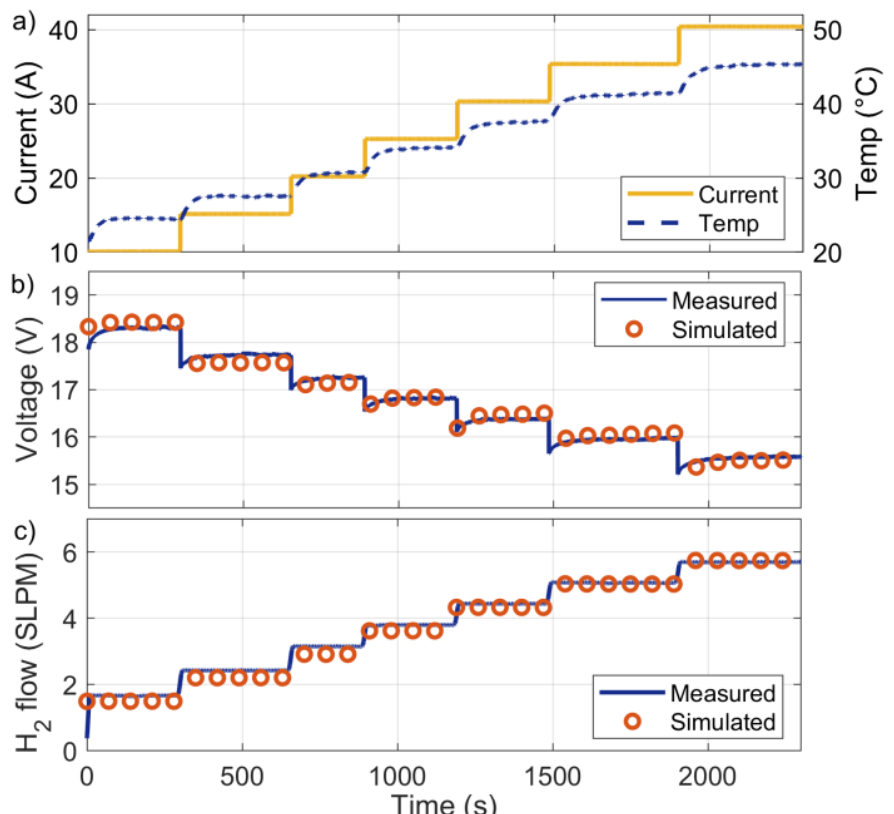

Fig. 8. The output voltage and hydrogen estimation analysis. a) The demanded current profile from the FC system and the corresponded stack temperature, $\mathrm{b}$ ) Online voltage estimation, and c) Comparison of $\mathrm{H} 2$ rate between the model and the real PEMFC. 


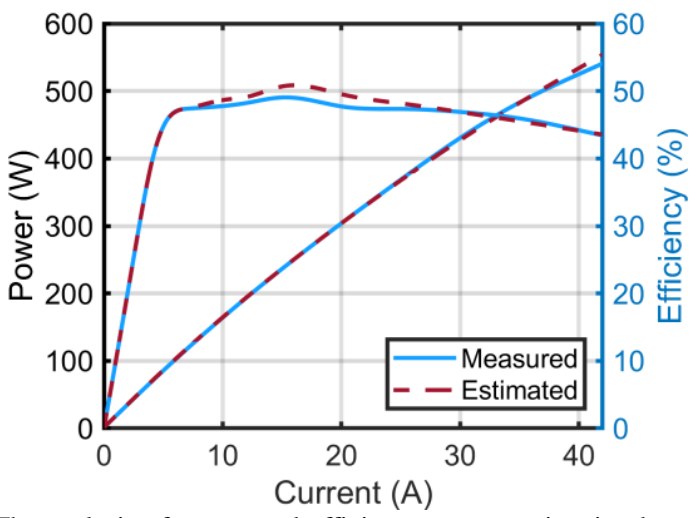

Fig. 9. The analysis of power and efficiency curves estimation by using the extracted parameters at $600 \mathrm{~s}$.

\section{Multi-mode fuzzy logic controller}

Fuzzy logic systems are designed to provide a number of strategic rules by means of linguistic labels. Several reasons, such as imprecise modeling of a vehicle's components, their nonlinear behavior, and the unknown behavior of exogenous factors, like traffic, weather, etc., can be counted for suitability of utilizing a FLC in EMS design of FCHEVs.

The proposed FLC has three inputs, which are demanded power, the derivation of the demanded power, and battery SOC. Choosing the requested power derivation as an input, besides the other two inputs, helps at using the PEMFC system in a more stable manner. The only output of the utilized FLC is the required power from the FC system. The initial input and output membership functions (MFs) are shown in Fig. 10. As is clear in this figure, the output MF is defined based on the ME and MP points of the FC system. The output of the FLC, which is between 0 and 1, goes under a defuzzification process to be transformed into a real quantifiable value. The defuzzification is done by utilizing a linear function in which the slope is defined by means of the estimated values of ME and MP points through the previously described online PEMFC modeling. In this way, as the FC system goes under degradation, the output MF is updated with respect to the real state of the FC system. Moreover, the distribution of the variables of the output MF stays the same though the transformation gains of the defuzzification process change. The fuzzy reasoning rules, shown in Fig. 11, are laid down based on the heuristic expertise. The initial MFs are also tuned heuristically and then improved by GA which is a metaheuristic approach. Since the optimization of fuzzy MFs is a classic method and is available in other similar works [7], its explanation has been considered redundant in this paper. The utilized objective function for performing the MFs adjustment is presented in (17). The constructing parameters of the input and output MFs, which come to 23 , are considered as decision variables for optimizing the FLC. To perform the optimization process, GA utilizes some natural procedures, such as crossover and mutation, to leave out the unfavorable populations and keep the most meritorious ones to create new generations. In this context, the process of survival of the fittest refers to the minimization trend of the defined objective function. The number of generations is set to 100 , the population size is 200 , the elite count is 10 , and the crossover fraction is 0.8 .

$$
\begin{aligned}
& \underset{\text { (params. })}{\min } J=w_{1} \sum_{j=1}^{K} m_{H 2}+w_{2} \sum_{j=1}^{K} N_{o n / o f f}+\$_{R P} \\
& P_{M F s_{k, \min }} \leq P_{M F s_{k}} \leq P_{M F s_{k, \max }}(\mathrm{k}=1 \ldots 23) \\
& M E \leq P_{F C} \leq M P \\
& 0.5 \leq S O C \leq 1
\end{aligned}
$$

Where $J$ is the objective function, $w_{1}$ is the cost conversion factor for hydrogen, $w_{2}$ the durability cost conversion factor, $m_{H 2}$ is the hydrogen consumption, $N_{\text {on/off }}$ is the number of on/off cycles in the PEMFC, $\$_{R P}$ is the cost of the recharge penalty (USD), $P_{M F S}$ is the parameter for defining the MFs, and $P_{F C}$ is the FC system power. The values of $w_{1}$ and $w_{2}$ are 2.3 $\mathrm{USD} / \mathrm{kg} \mathrm{H} 2$ and $0.032 \mathrm{USD}$ respectively. These values have been defined based on the 2020 technical targets put forward by the U.S. Department of Energy in the Multi-Year Research, Development, and Demonstration Plan. The optimization process of the fuzzy controllers has been done by considering a recharge penalty step $\left(\$_{R P}\right)$ at the end of each profile. In this way, the battery is fully recharged by using the maximum power point of the PEMFC stack at the end of each test and the USD cost of the additional required hydrogen is added to the total cost function. It should be noted that the recharge step is performed by setting the stack on its maximum power to punish the strategy if it finishes in low SOC level.
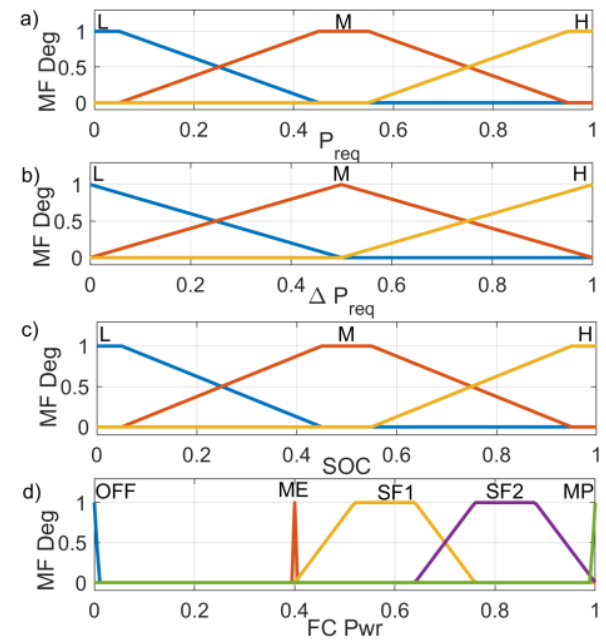

Fig. 10. The primary distribution of the input and output MFs, a) Input 1: requested power, b) Input 2: requested power derivation, c) Input 3: battery SOC, and d) Output: reference power of the FC system.

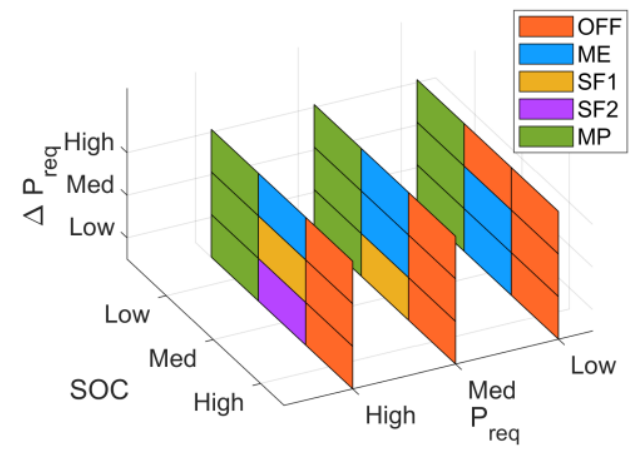

Fig. 11. The designed FLC rule base. (Fuzzy system: Mamdani, Inference mechanism: AND (minimum operator), and diffuzication: centroid). The output MF labels are shown in the legend of this figure. 
The multi-mode controller should be developed in a way to embrace all the traffic conditions since it is supposed to work online without a prior knowledge of the driving cycle. As the driving data are clustered into three classes by the developed SOM recognizer, one optimized FLC needs to be developed for each class and then the three controllers should be put together to form the multi-mode FLC. Each optimized FLC can be considered as the near optimal controller for each class of the driving data. However, due to the high volume of data that each cluster contains, the optimization process would be very timeconsuming for all the driving data. In this regard, a representative driving cycle is developed for each of the driving profile to reduce the required time for optimization process. Fig. 12 shows the extracted representative driving cycles, namely low-speed, medium-speed, and high-speed, and their average velocities. These driving cycles are used as the input driving data for the process of FLC optimization. Each of them is composed of the nearest micro-trips to the center of cluster they belong to. The cluster centers have been already calculated by the developed SOM. The distance between each cluster center and each micro-trip is calculated by the Euclidean distance $(e d(x, y))$ as:

$$
e d(x, y)=\sqrt{\sum_{i=1}^{n}\left(x_{i}-y_{i}\right)^{2}}
$$

where $x=\left(x_{1}, x_{2}, \ldots, x_{10}\right)$ is the cluster center vector, $y=$ $\left(y_{1}, y_{2}, \ldots, y_{10}\right)$ is the micro-trip vector, and $n$ is the number of driving features, which is 10 in this work. There are three cluster center vectors and 130 micro-trip vectors where each vector contains 10 elements. The distance between each cluster center and all the micro-trips is calculated and then the nearest micro-trips to the center are combined to reach an almost 2000$\mathrm{s}$ representative cycle for every cluster. As explained before, the defuzzification function of the output MF is updated when a noticeable drift is observed in the maximum operating points of the FC system, which are available from the online semiempirical model.

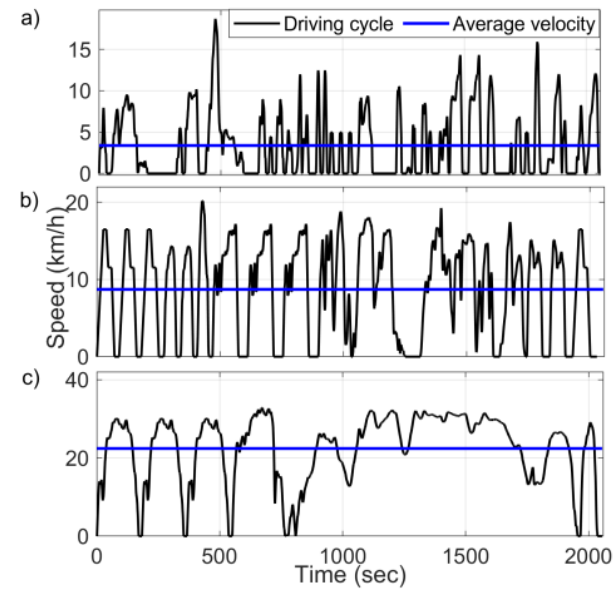

Fig. 12. The extracted representative driving cycles, a) Low-speed profile, b) Medium-speed profile, and c) High-speed profile.

\section{Primary evaluation of the proposed strategy}

To have a primary assessment of the developed multi-mode controller and the SOM classifier, a comparative analysis, in terms of the costs of $\mathrm{H} 2$, ON/OFF cycles, final SOC recharge penalty, and the total cost, is performed for five cases, as explained further in this section. The main purpose of the first three cases is to examine the performance of the SOM classifier of the proposed online multi-mode EMS regarding unnecessary switches. Case 4 is a real challenge between the proposed multimode EMS and one optimized fuzzy controller. Finally, Case 5 evaluates the performance of the proposed EMS under a new driving condition and compares it with two other strategies.

1) Case 1: low-speed (LS) driving profile (Fig. 12a)

In this case, the performance of the online multi-mode EMS is compared with only the first mode of the controller (FLC1), which is an offline optimized single-fuzzy EMS specifically designed for LS driving profile. According to Table VI, the performance of the both controllers is completely the same indicating the SOM classifier has used the correct mode.

2) Case 2: medium-speed (MS) driving profile (Fig. 12b)

The second case compares the performance of the online multi-mode EMS with the second mode of the controller (FLC2), which is an offline optimized single-fuzzy EMS specifically designed for MS driving profile. Table VI shows that the total cost of the online multi-mode EMS is $2 \%$ more than the FLC2, which can be owing to very few switches in the recognition process.

3) Case 3: high-speed (HS) driving profile (Fig. 12c)

This case compares the performance of the online multimode EMS with the third mode of the controller (FLC3), which is an offline optimized single-fuzzy EMS specifically designed for HS driving profile. Table VI indicates that the proposed EMS can closely approach the optimized results obtained by FLC3 with less than one percent difference in the total cost. Obviously, the proposed EMS does not have unnecessary switches for determining the mode of operation.

4) Case 4: representative (Rep.) driving profile

This case compares the performance of the online multimode EMS with an offline optimized single-fuzzy EMS (Opt. for rep.) particularly designed for the Rep. driving profile. The Rep. driving profile is a concatenation of LS, MS, and HS profiles. The online multi-mode EMS has three optimized FLCs corresponding to each of LS, MS, and HS conditions inside the Rep. driving profile while the Opt. for rep. EMS is only one optimized FLC for the whole driving profile. According to Table VI, it can be seen that the online multi-mode EMS outperforms the Opt. for rep. EMS by 3.7 percent in terms of the total cost. This superior performance shows the applicability of the proposed multimode EMS.

5) Case 5: combined driving profile (Fig. 7)

So far, all the discussed cases have been done by using the known driving profiles. However, in this case study, to better clarify the effectiveness and flexibility of the online multi-mode controller in real-time unknown driving conditions, its performance is compared with the Opt. for rep. EMS under combined driving profile, which is a new driving condition for both of the strategies. Moreover, the performance of the proposed strategy is compared with one of the commonly used real-time strategies in the literature called bounded load following strategy (BLFS) [39, 45]. 
BLFS is a hysteresis based energy management to split the power among the components of a fuel cell hybrid electric vehicle. BLFS normally provides three modes of operation for the PEMFC stack including ON/OFF, $P_{F C_{-} \text {min }}$, and $P_{F C_{-} \max }$ with respect to the battery SOC level. To assure a low hydrogen consumption, the maximum efficiency of the PEMFC stack is selected as the $P_{F C_{-} \min }$ mode. This choice stems from the fact that the hydrogen consumption and the degradation of the stack is higher within the open circuit voltage and the best efficiency point area of the PEMFC. Therefore, when the PEMFC is turned on, the ME mode is activated. $P_{F C_{-} \max }$ mode, which sets the stack on its maximum power, is triggered when the battery SOC reaches the minimum SOC level. Furthermore, the $P_{F C}$ max mode assists the battery pack in high traction power operations. The battery SOC can fluctuate between $45 \%$ and $95 \%$ $(0.45 \leq S O C \leq 95)$ [39]. As reported by Table VI, the proposed multi-mode EMS outperforms the Opt. for rep. and the BLFS strategies by almost 7 percent. This performance distinction indicates that the proposed EMS performs well when confronting new driving conditions.

TABLE VI

COMPARISON OF THE MULTI-MODE CONTROLLER AND THE OPTIMIZED CONTROLLER OF EACH REPRESENTATIVE DRIVING CYCLE

\begin{tabular}{|c|c|c|c|c|c|}
\hline Case study & Cost (USD) & $\mathrm{H}_{2}$ & $\begin{array}{c}\text { ON/OFF } \\
\text { cycles }\end{array}$ & $\begin{array}{c}\text { Recharge } \\
\text { penalty }\end{array}$ & Total \\
\hline Case 1 & $\begin{array}{l}\text { Opt. for LS } \\
\text { Multi-mode }\end{array}$ & $\begin{array}{l}12.49 \\
12,49\end{array}$ & $\begin{array}{l}0 \\
0\end{array}$ & $\begin{array}{l}8.21 \\
8.21\end{array}$ & $\begin{array}{l}20.70 \\
20.70\end{array}$ \\
\hline Case 2 & $\begin{array}{l}\text { Opt. for MS } \\
\text { Multi-mode }\end{array}$ & $\begin{array}{l}12.72 \\
13.25\end{array}$ & $\begin{array}{l}0 \\
0\end{array}$ & $\begin{array}{l}10.55 \\
10.51\end{array}$ & $\begin{array}{l}23.27 \\
23.76\end{array}$ \\
\hline Case 3 & $\begin{array}{l}\text { Opt. for HS } \\
\text { Multi-mode }\end{array}$ & $\begin{array}{l}13.67 \\
14.15\end{array}$ & $\begin{array}{l}0 \\
0\end{array}$ & $\begin{array}{l}18.01 \\
17.66\end{array}$ & $\begin{array}{l}31.67 \\
31.81\end{array}$ \\
\hline Case 4 & $\begin{array}{l}\text { Opt. for rep. } \\
\text { Multi-mode }\end{array}$ & $\begin{array}{l}35.64 \\
34.16\end{array}$ & $\begin{array}{l}2.97 \\
2.78\end{array}$ & $\begin{array}{l}2.38 \\
2.52\end{array}$ & $\begin{array}{c}41 \\
39.48\end{array}$ \\
\hline Case 5 & $\begin{array}{l}\text { Opt. for rep. } \\
\text { Multi-mode } \\
\text { BLFS }\end{array}$ & $\begin{array}{l}28.05 \\
25.66 \\
28.76\end{array}$ & $\begin{array}{l}0 \\
0 \\
0\end{array}$ & $\begin{array}{l}3.84 \\
3.92 \\
3.00\end{array}$ & $\begin{array}{l}31.90 \\
29.58 \\
31.76\end{array}$ \\
\hline
\end{tabular}

\section{EXPERIMENT AND RESULTS ANALYSIS}

The performance of the proposed online multi-mode EMS is comprehensively studied in this section. In this respect, two principal scenarios are taken into consideration. Both of these scenarios have been implemented on the developed test bench to have realistic perception of the FCHEV performance.

In the first scenario, the combined driving cycle, introduced in section 2, is imposed to the vehicle as the input and the performance of the proposed EMS is compared with the offlineoptimized EMS in terms of hydrogen consumption and efficient use of the energy sources. The new FC system is used throughout the first scenario. Fig. 13 presents the obtained results from the performed test in scenario 1. Fig. 13a shows the traction power, obtained from imposing the combined driving cycle to the system, the supplied power by FC system, and the battery pack for the case of offline optimized EMS. It should be noted that this offline strategy knows the driving cycle in advanced as opposed to the online multi-mode EMS. Fig. 13b indicates the traction power and its split between the FC and the battery pack for the case of online multi-mode strategy. From these two figures, it is clear that the FC system is being used to supply the main portion of the requested power in a stable manner, compared to the battery, which is mostly responsible for absorbing the fast transitions. Fig. 13c compares the battery SOC of the optimal and multi-mode controllers. It is obvious that the optimal strategy keeps the SOC in a higher level due to its priory knowledge about requested power. Fig. 13d presents the distribution of the FC operating points while meeting the requested power. Form Fig. 13d, it is clear that the proposed strategy is capable of limiting the operation of the FC system within the safe zone, which is between MP and ME, and tends to operate the FC system in the high efficient zone, which is around $15 \mathrm{~A}$, most of the time.
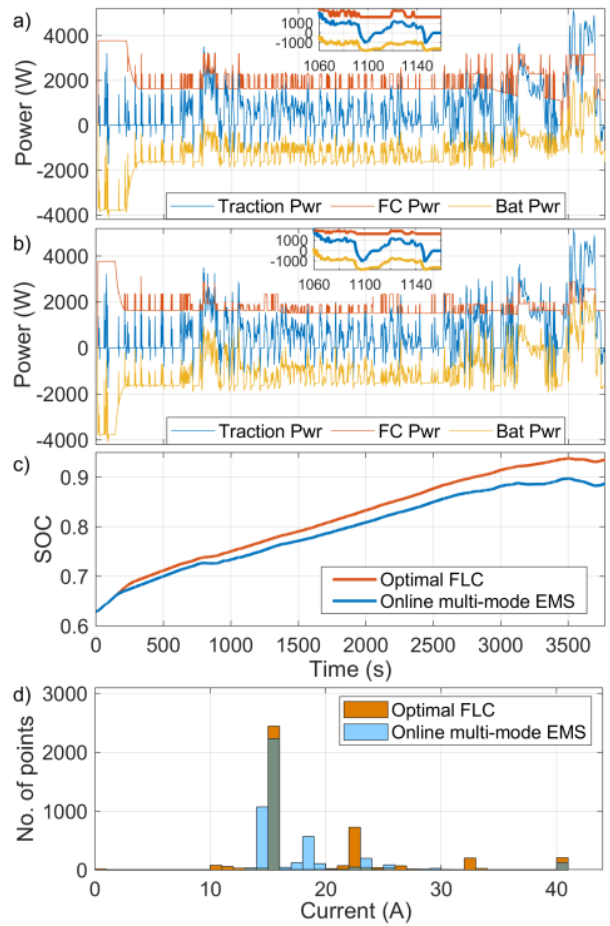

Fig. 13. Scenario 1 for evaluation of the proposed multi-mode EMS, a) Power split by the optimal FLC, b) Power split by the online multi-mode strategy, c) Battery SOC comparison of the two strategies, and d) PEMFC operating points distribution.

In the second scenario, the capability of the proposed EMS to deal with the FC system performance drifts is scrutinized. In this respect, the EMS test with the combined driving cycle is repeated, but by using the old PEMFC. Moreover, to signify the importance of the online PEMFC characteristics tracking, once the test is performed by deactivating the online identification, and the second time it is done by activating it. Fig. 14 shows the obtained outcomes of these tests. Fig. 14a and Fig. 14b represent the power split for offline and online multi-mode EMSs respectively. According to Fig. 14a, the offline multimode strategy experiences a lot of start-ups and shutdowns in the first $400 \mathrm{~s}$ in the FC system as it tries to recharge the battery by using the PEMFC in high power. However, it is not aware of the fact that the FC system has been degraded and its MP and ME points have changed. Therefore, it demands for a power 
level that is out of the ability of the FC to supply and causes these on/off cycles.

On the other hand, in case of the proposed online EMS as shown in Fig. 14b, in the first $100 \mathrm{~s}$, the identification is performed to realize the real characteristics of the FC system and update the defuzzification tuning of the controller. After that, the FC system works in the high power area to recharge the battery pack to a certain level in addition to supplying the requested power without having any on/off cycles. Fig. 14c compares the SOC level of the battery for both cases and Fig. 14d demonstrates the FC system operating points distribution. From Fig. 14d, it is clear that the online multi-mode EMS utilizes the FC system more efficiently which can prolong its lifetime besides improving the fuel economy of the vehicle. Table VII compares the obtained cost by each of the EMSs in both scenarios. According to this table, the proposed online multimode strategy shows a very close performance to the optimal FLC in scenario 1. This close performance demonstrates that the proposed online EMS is able to handle unknown driving conditions with an acceptable fuel economy. The presented results of scenario 2 also confirms the satisfactory adaptation of the proposed EMS to the performance drifts of the FC system, which is a distinguishing feature of this suggested EMS. This adaptation to the real state of the FC system has made $8 \%$ of performance improvement in the online multi-mode strategy in scenario 2 .
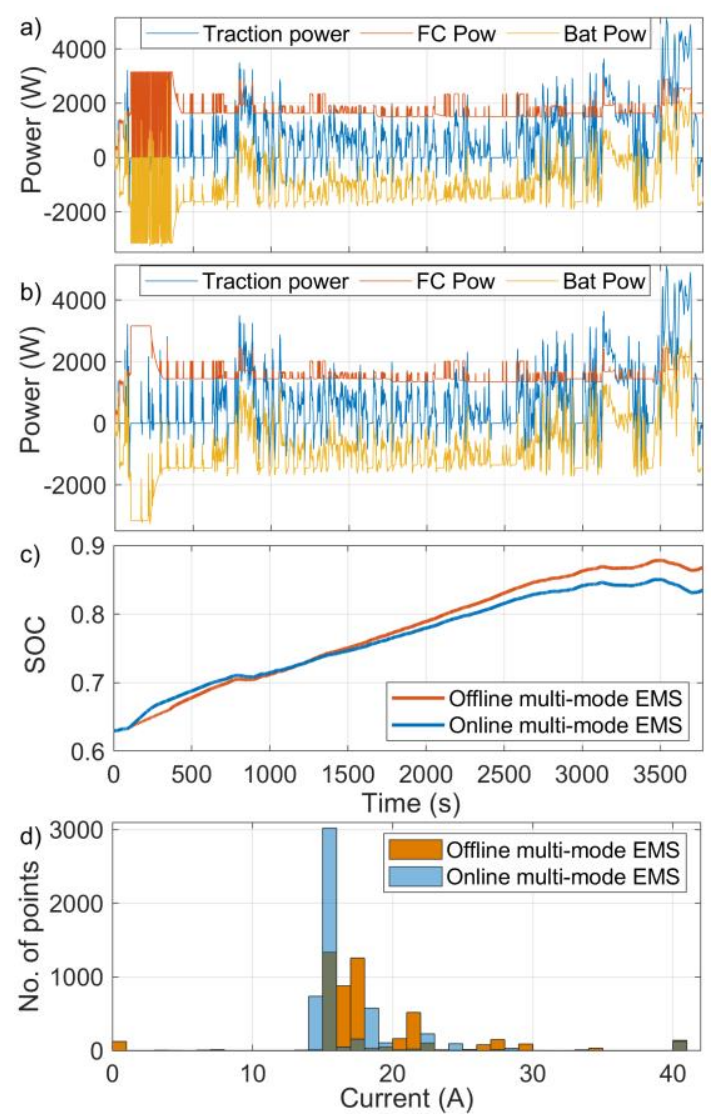

Fig. 14. Scenario 2 for assessing the online performance tracking of the FC system significance in the proposed multi-mode EMS, a) Power split by the online multi-mode EMS, b) Power split by the offline multi-mode strategy, c) Battery SOC comparison of the strategies, and d) PEMFC operating points distribution.

TABLE VII

The cost comparison of the EMSs in the two performed scenarios

\begin{tabular}{ccccc}
\hline \hline & \multicolumn{2}{c}{ Scenario 1 } & \multicolumn{2}{c}{ Scenario 2 } \\
\cline { 2 - 5 } Cost (USD) & Optimized & Online & Offline & Online \\
mul & Multi-mode & multi-mode & multi-mode \\
$\mathrm{H}_{2}$ & 28.82 & 26.96 & 28.92 & 25.56 \\
$\begin{array}{c}\text { ON/OFF } \\
\text { cycles }\end{array}$ & 0 & 0 & 0.80 & 0 \\
$\begin{array}{c}\text { Recharge } \\
\text { penalty }\end{array}$ & 13 & 6.00 & 7.98 & 8.99 \\
Total & 32.16 & 32.96 & 37.70 & 34.55 \\
\hline \hline
\end{tabular}

\section{CONCLUSION}

This paper presents a new online multi-mode EMS for a FCHEV. This EMS is mainly composed of a SOM based driving condition classifier and a multi-model FLC. The FLC output MF is constantly adjusted based on the online estimation of the FC system MP and ME boundaries by KF and a semiempirical PEMFC model. The developed SOM recognizes the driving condition and activates the most proper mode of the FLC at each update to efficiently supply the request power from the vehicle. The performance of the proposed online strategy is compared with an offline optimized FLC under a combined driving cycle of CYC_NewYorkBus, CYC_UDDS, and WLTC_class 3 and a satisfactory result is obtained with only a two-percent difference in terms of the total cost of hydrogen consumption and on/off cycles of the FC system. Moreover, the performance of the proposed EMS is tested when the FC system undergoes a sixteen-percent drift regarding the maximum power. In this case, the proposed online EMS adapts to the real state of the FC system and improves the performance of the vehicle by eight percent compared to the offline multi-mode controller.

Although this work has well established the potential of the proposed online EMS, some prospects for extending the scope of this paper remain as follows:

- Incorporating an online battery management system into the presented strategy to reach a holistic EMS.

- Performing a lifetime and ageing study of the energy sources under the proposed EMS.

\section{REFERENCES}

[1]
W. R. Black and N. Sato, "From Global Warming to Sustainable Transport 1989-2006," International Journal of Sustainable Transportation, vol. 1, pp. 73-89, 2007/05/21 2007.

A. Khaligh and Z. Li, "Battery, Ultracapacitor, Fuel Cell, and Hybrid Energy Storage Systems for Electric, Hybrid Electric, Fuel Cell, and Plug-In Hybrid Electric Vehicles: State of the Art," IEEE Transactions on Vehicular Technology, vol. 59, pp. 2806-2814, 2010.

S. Samuelsen, "The automotive future belongs to fuel cells range, adaptability, and refueling time will ultimately put hydrogen fuel cells ahead of batteries," IEEE Spectrum, vol. 54, pp. 38-43, 2017. N. Sulaiman, M. A. Hannan, A. Mohamed, E. H. Majlan, and W. R. Wan Daud, "A review on energy management system for fuel cell hybrid electric vehicle: Issues and challenges," Renewable and Sustainable Energy Reviews, vol. 52, pp. 802-814, 2015/12/01/ 2015.

Y. Huang, H. Wang, A. Khajepour, B. Li, J. Ji, K. Zhao, et al., "A review of power management strategies and component sizing methods for hybrid vehicles," Renewable and Sustainable Energy Reviews, vol. 96, pp. 132-144, 2018/11/01/ 2018. 
[6] S. F. Tie and C. W. Tan, "A review of energy sources and energy management system in electric vehicles," Renewable and Sustainable Energy Reviews, vol. 20, pp. 82-102, 2013/04/01/ 2013.

[7] R. Zhang and J. Tao, "GA-Based Fuzzy Energy Management System for FC/SC-Powered HEV Considering H2 Consumption and Load Variation," IEEE Transactions on Fuzzy Systems, vol. 26, pp. 1833-1843, 2018.

[8] M. Kandi-D, M. Soleymani, and A. A. Ghadimi, "Designing an Optimal Fuzzy Controller for a Fuel Cell Vehicle Considering Driving Patterns," Scientia Iranica, vol. 23, pp. 218-227, 2016.

[9] J. Han, D. Kum, and Y. Park, "Synthesis of Predictive Equivalent Consumption Minimization Strategy for Hybrid Electric Vehicles Based on Closed-Form Solution of Optimal Equivalence Factor," IEEE Transactions on Vehicular Technology, vol. 66, pp. 56045616, 2017.

[10] Y. Ren and Z. Wu, "Research on the Energy Management Strategy of Hybrid Vehicle Based on Pontryagin's Minimum Principle," in 2018 10th International Conference on Intelligent Human-Machine Systems and Cybernetics (IHMSC), 2018, pp. 356-361.

[11] A. Rezaei, J. B. Burl, and B. Zhou, "Estimation of the ECMS Equivalent Factor Bounds for Hybrid Electric Vehicles," IEEE Transactions on Control Systems Technology, vol. 26, pp. 2198$2205,2018$.

[12] C. Zheng, W. Li, and Q. Liang, "An Energy Management Strategy of Hybrid Energy Storage Systems for Electric Vehicle Applications," IEEE Transactions on Sustainable Energy, vol. 9, pp. 1880-1888, 2018.

[13] Z. Chen, R. Xiong, and J. Cao, "Particle swarm optimization-based optimal power management of plug-in hybrid electric vehicles considering uncertain driving conditions," Energy, vol. 96, pp. 197208, 2016/02/01/ 2016.

[14] A. Ali and D. Söffker, "Towards Optimal Power Management of Hybrid Electric Vehicles in Real-Time: A Review on Methods, Challenges, and State-Of-The-Art Solutions," Energies, vol. 11, p. 476, 2018.

[15] H. Kazemi, Y. P. Fallah, A. Nix, and S. Wayne, "Predictive AECMS by Utilization of Intelligent Transportation Systems for Hybrid Electric Vehicle Powertrain Control," IEEE Transactions on Intelligent Vehicles, vol. 2, pp. 75-84, 2017.

[16] H. S. Ramadan, M. Becherif, and F. Claude, "Energy Management Improvement of Hybrid Electric Vehicles via Combined GPS/RuleBased Methodology," IEEE Transactions on Automation Science and Engineering, vol. 14, pp. 586-597, 2017.

[17] E. Kamal and L. Adouane, "Intelligent Energy Management Strategy Based on Artificial Neural Fuzzy for Hybrid Vehicle," IEEE Transactions on Intelligent Vehicles, vol. 3, pp. 112-125, 2018.

[18] C. M. Martinez, X. Hu, D. Cao, E. Velenis, B. Gao, and M. Wellers, "Energy Management in Plug-in Hybrid Electric Vehicles: Recent Progress and a Connected Vehicles Perspective," IEEE Transactions on Vehicular Technology, vol. 66, pp. 4534-4549, 2017.

[19] A. Fotouhi, R. Yusof, R. Rahmani, S. Mekhilef, and N. Shateri, "A review on the applications of driving data and traffic information for vehicles' energy conservation," Renewable and Sustainable Energy Reviews, vol. 37, pp. 822-833, 2014/09/01/ 2014.

[20] M. Kandi Dayeni and M. Soleymani, "Intelligent energy management of a fuel cell vehicle based on traffic condition recognition," Clean Technologies and Environmental Policy, vol. 18, pp. 1945-1960, 2016/08/01 2016.

[21] Z. Chen, C. C. Mi, J. Xu, X. Gong, and C. You, "Energy Management for a Power-Split Plug-in Hybrid Electric Vehicle Based on Dynamic Programming and Neural Networks," IEEE Transactions on Vehicular Technology, vol. 63, pp. 1567-1580, 2014.

[22] Y. L. Murphey, J. Park, Z. Chen, M. L. Kuang, M. A. Masrur, and A. M. Phillips, "Intelligent Hybrid Vehicle Power Control—Part I: Machine Learning of Optimal Vehicle Power," IEEE Transactions on Vehicular Technology, vol. 61, pp. 3519-3530, 2012.

[23] Z. Chen, L. Li, B. Yan, C. Yang, C. M. Martínez, and D. Cao, "Multimode Energy Management for Plug-In Hybrid Electric Buses Based on Driving Cycles Prediction," IEEE Transactions on Intelligent Transportation Systems, vol. 17, pp. 2811-2821, 2016.
[24] R. Zhang, J. Tao, and H. Zhou, "Fuzzy optimal energy management for fuel cell and supercapacitor systems using neural network based driving pattern recognition," IEEE Transactions on Fuzzy Systems, pp. 1-1, 2018.

[25] J. Liu, Y. Chen, J. Zhan, and F. Shang, "An On-Line Energy Management Strategy Based on Trip Condition Prediction for Commuter Plug-In Hybrid Electric Vehicles," IEEE Transactions on Vehicular Technology, vol. 67, pp. 3767-3781, 2018.

[26] H. Lim and W. Su, "Hierarchical Energy Management for PowerSplit Plug-In HEVs Using Distance-Based Optimized Speed and SOC Profiles," IEEE Transactions on Vehicular Technology, vol. 67, pp. 9312-9323, 2018.

[27] H. Tian, X. Wang, Z. Lu, Y. Huang, and G. Tian, "Adaptive Fuzzy Logic Energy Management Strategy Based on Reasonable SOC Reference Curve for Online Control of Plug-in Hybrid Electric City Bus," IEEE Transactions on Intelligent Transportation Systems, vol. 19, pp. 1607-1617, 2018.

[28] K. Song, F. Li, X. Hu, L. He, W. Niu, S. Lu, et al., "Multi-mode energy management strategy for fuel cell electric vehicles based on driving pattern identification using learning vector quantization neural network algorithm," Journal of Power Sources, vol. 389, pp. 230-239, 2018/06/15/ 2018.

[29] D. Zhou, A. Al-Durra, F. Gao, A. Ravey, I. Matraji, and M. Godoy Simões, "Online energy management strategy of fuel cell hybrid electric vehicles based on data fusion approach," Journal of Power Sources, vol. 366, pp. 278-291, 2017/10/31/2017.

[30] J. Chen, C. Xu, C. Wu, and W. Xu, "Adaptive Fuzzy Logic Control of Fuel-Cell-Battery Hybrid Systems for Electric Vehicles," IEEE Transactions on Industrial Informatics, vol. 14, pp. 292-300, 2018.

[31] M. Kandidayeni, A. Macias, A. A. Amamou, L. Boulon, S. Kelouwani, and H. Chaoui, "Overview and benchmark analysis of fuel cell parameters estimation for energy management purposes," Journal of Power Sources, vol. 380, pp. 92-104, 2018/03/15/ 2018. [32] M. Kandidayeni, A. Macias, A. A. Amamou, L. Boulon, and S. Kelouwani, "Comparative Analysis of Two Online Identification Algorithms in a Fuel Cell System," Fuel Cells, vol. 18, pp. 347-358, 2018.

[33] N. Bizon and P. Thounthong, "Real-time strategies to optimize the fueling of the fuel cell hybrid power source: A review of issues, challenges and a new approach," Renewable and Sustainable Energy Reviews, vol. 91, pp. 1089-1102, 2018/08/01/ 2018.

[34] D. Zhou, A. Al-Durra, I. Matraji, A. Ravey, and F. Gao, "Online Energy Management Strategy of Fuel Cell Hybrid Electric Vehicles: A Fractional-Order Extremum Seeking Method," IEEE Transactions on Industrial Electronics, vol. 65, pp. 6787-6799, 2018.

[35] D. Zhou, A. Ravey, A. Al-Durra, and F. Gao, "A comparative study of extremum seeking methods applied to online energy management strategy of fuel cell hybrid electric vehicles," Energy Conversion and Management, vol. 151, pp. 778-790, 2017/11/01/ 2017.

[36] K. Ettihir, M. Higuita Cano, L. Boulon, and K. Agbossou, "Design of an adaptive EMS for fuel cell vehicles," International Journal of Hydrogen Energy, vol. 42, pp. 1481-1489, 2017/01/12/ 2017.

[37] K. Ettihir, L. Boulon, and K. Agbossou, "Optimization-based energy management strategy for a fuel cell/battery hybrid power system," Applied Energy, vol. 163, pp. 142-153, 2016/02/01/ 2016.

[38] L. Xu, J. Li, J. Hua, X. Li, and M. Ouyang, "Adaptive supervisory control strategy of a fuel cell/battery-powered city bus," Journal of Power Sources, vol. 194, pp. 360-368, 2009/10/20/ 2009.

[39] M. Carignano, V. Roda, R. Costa-Castelló, L. Valiño, A. Lozano, and F. Barreras, "Assessment of Energy Management in a Fuel Cell/Battery Hybrid Vehicle," IEEE Access, vol. 7, pp. 1611016122, 2019.

[40] V. H. Johnson, "Battery performance models in ADVISOR," Journal of Power Sources, vol. 110, pp. 321-329, 2002/08/22/2002.

[41] R. F. Mann, J. C. Amphlett, M. A. I. Hooper, H. M. Jensen, B. A. Peppley, and P. R. Roberge, "Development and application of a generalised steady-state electrochemical model for a PEM fuel cell," Journal of Power Sources, vol. 86, pp. 173-180, 2000/03/01/2000.

[42] J. P. F. Trovão, M. Roux, M. É, and M. R. Dubois, "Energy- and Power-Split Management of Dual Energy Storage System for a Three-Wheel Electric Vehicle," IEEE Transactions on Vehicular Technology, vol. 66, pp. 5540-5550, 2017. 
T. Kohonen, E. Oja, O. Simula, A. Visa, and J. Kangas, "Engineering applications of the self-organizing map," Proceedings of the IEEE, vol. 84, pp. 1358-1384, 1996.

[44] S. S. Haykin, Kalman Filtering and Neural Networks: John Wiley I\&amp; Sons, Inc., 2001.

[45] K. Ettihir, L. Boulon, and K. Agbossou, "Energy management strategy for a fuel cell hybrid vehicle based on maximum efficiency and maximum power identification," IET Electrical Systems in Transportation, vol. 6, pp. 261-268, 2016.

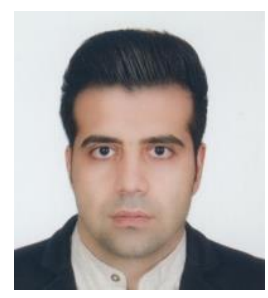

Mohsen Kandidayeni ( $\left.\mathrm{S}^{\prime} 18\right)$ is currently a $\mathrm{PhD}$ student at Université du Québec à Trois-Rivières (UQTR), working on energy-related topics such as Hybrid Electric Vehicles, Fuel cell systems, Energy management, Multiphysics systems, Modeling and Control. He graduated from Arak University with a master's degree in Mechatronics in 2014. His research interests are in the area of Renewable Energy, Fuel Cell, Transportation, Intelligent Transport Systems, Vehicular Systems Control, Hybrid Electric and Electric Vehicles.

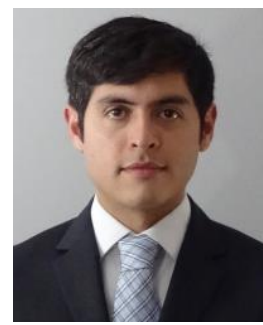

Alvaro Macias F. (S'17) was born in Mexico City, in 1992. He received the B.S. degree in mechatronics engineering from Tec de Monterrey, Guadalajara, Mexico, in 2015 and the M.S. degree in electrical engineering from Université du Québec à TroisRivières, Canada, in 2018. He is currently pursuing the Ph.D. degree in electrical engineering at Université du Québec à Trois-Rivières, Canada. From 2015 to 2016, he worked as Research and Development in the Centro de Investigación y de Estudios Avanzados del Instituto Politécnico Nacional, Mexico. His current research interest includes the development of energy management strategies for fuel cell systems, passive and active system configuration, and fuel cell modeling.

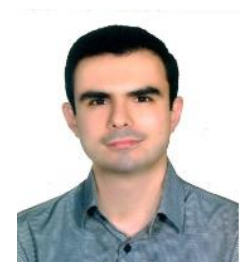

Arash Khalatbarisoltani (S'18) received his Bachelor's degree in Electrical Engineering from Sadjad University of Technology, Mashhad, Iran, 2012, and his Master's degree in Mechatronics from Arak University, Arak, Iran, 2016. He is currently a $\mathrm{PhD}$ student with the department of Electrical and Computer Engineering at Université du Québec à Trois-Rivières (UQTR) in the Hydrogen Research Institute (IRH). His research interests are decentralized systems, fuel cell systems, energy management, fuel cell Vehicles, and modular systems. His work deals with optimization, energy management of multi power source systems, modular systems, game theory, and decentralized control.

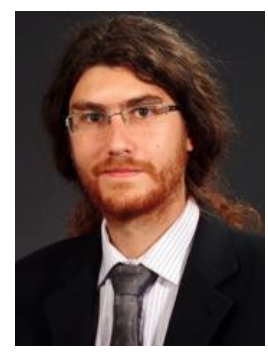

Loïc Boulon (M'10, SM'16) received the master's degree in electrical and automatic control engineering from the University of Lille (France), in 2006. Then, he obtained a $\mathrm{PhD}$ in electrical engineering from University of Franche-Comté (France). Since 2010, he is a professor at Université du Québec à TroisRivières (Canada) and he works at the Hydrogen Research Institute (Full Professor since 2016). His work deals with modeling, control and energy management of multiphysics systems. His research interests include hybrid electric vehicles, energy and power sources (especially battery in cold weather operation), and fuel cell systems. He has published more than 100 scientific papers in peer-reviewed international journals and international conferences. In 2015, Loïc Boulon was general chair of the IEEE-Vehicular Power and Propulsion Conference in Montréal (QC, Canada). Prof. Loïc Boulon is VP-Motor Vehicles of the IEEE Vehicular Technology Society and he is the holder of the Canada Research Chair in Energy Sources for the Vehicles of the future.

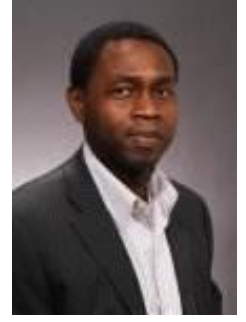

Sousso Kelouwani (M'00-SM'17) received the B.S. and the M.Sc.A. degrees from Université du Québec à Trois-Rivières, Trois-Rivières, QC, Canada, in 2000 and 2002, respectively, and the Ph.D. degree (automation and systems) from École Polytechnique de Montréal, Montréal, QC, Canada, in 2010, all in electrical engineering. Holder of the Industrial Research Chair DIVEL in Intelligent Navigation of Autonomous Industrial Vehicles, Sousso Kelouwani is a full professor of Mechatronics at the Department of Mechanical Engineering at UQTR (since 2018). Before starting his doctoral studies, he worked in research and development in the field of cell phone application optimization for Cilys 53 inc. (2002-2005) and Openwave inc. (2005-2006). Holder of three patents in the United States, his research interest focuses on the optimization of energy systems for vehicular applications, advanced driving assistance techniques, eco-energy navigation of autonomous vehicles, hybridization of energy sources for vehicles with low ecological impact (battery, fuel cell, hydrogen generator, etc.) in harsh weather conditions. In 2017, he received the Environment Award of the Grand Prix for Excellence in Transport from the Quebec Transportation Association (AQTr) for the development of a hydrogen range extender based on a hydrogen generator for electric vehicles. He was also a recipient of the Canada Governor General's Gold Medal in 2000. Moreover, he has worked with several Canadian transportation companies to develop intelligent, energy-efficient and driverless vehicles.

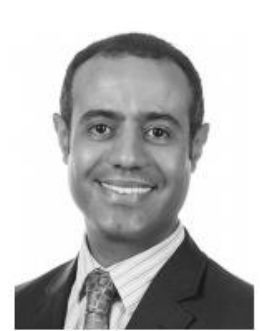

Hicham Chaoui (S'01-M'12-SM'13) received the $\mathrm{Ph} . \mathrm{D}$. degree in electrical engineering (with honors) from the University of Quebec, Trois-Rivières, QC, Canada, in 2012. His career has spanned both academia and industry in the field of control and energy systems. From 2007 to 2014, he held various engineering and management positions in the Canadian industry. From 2014 to 2016, he was an Assistant Professor at Tennessee Technological University, Cookeville, TN, USA. Since then, he has been a Faculty Member at Carleton University, Ottawa, ON, Canada. His research interests include adaptive and nonlinear control theory, intelligent control, robotics, electric motor drives, and energy conversion and storage systems. His scholarly work has produced more than 100 journal and conference publications. Dr. Chaoui was a recipient of the Best Thesis Award and the Governor General of Canada Gold Medal Award. He is a senior member of IEEE and a registered professional engineer in the province of Ontario. He is also an Associate Editor of IEEE Transactions on Vehicular Technology. 